Disclosure of Interest: None declared

DOI: 10.1136/annrheumdis-2017-eular.4316

\section{SAT0727-HPR THE EFFECT OF INSPIRATORY MUSCLE TRAINING ON AEROBIC CAPACITY, PULMONARY FUNCTION AND FUNCTIONAL STATUS IN PATIENTS WITH ANKYLOSING SPONDYLITIS: A RANDOMIZED CONTROLLED STUDY}

B. Basakci Calik, E. Gür Kabul, H. Taskın, O. Telli Atalay, U. Bas Aslan, M. Tascı, F. Bıcakcı, A.I. Yıldız. Pamukkale University, Denizli, Turkey

Background: In ankylosing spondylitis (AS) the chronic inflammatory process mainly affects the axial skeleton with ensuing pain and limitation of thoracic and spinal mobility. Most of the AS patients have the complaint of reduced exercise capacity. Pulmonary function impairment, chest wall restriction, weak respiratory muscle performance, peripheral muscle weakness and deconditioning have been reported as the hypotheses for reduced exercise capacity.

Objectives: The aim of this study was to evaluate the effects of inspiratory muscle training on aerobic capacity, pulmonary function and functional status in patients with AS.

Methods: A total of 32 patients (18 female, 16 male; mean age: $37.3 \pm 10.4$ years) were included in this study. The patients were randomized as two groups; Group I consisted of 16 patients, received inspiratory muscle training (IMT) in addition to conventional exercise (CE), Group II consisted of 16 patients, received only CE. All assessments were done before and after the training ( 8 weeks) for every patient. Standard pulmonary function tests were applied for pulmonary volumes. Maximum inspiratory pressure (PImax), maximum expiratory pressure (PEmax) were also measured as respiratory muscle strength. Six-minute walk test (6MWT) was used for the assessment of aerobic capacity. For the evaluation of functional status, the Bath Ankylosing Spondylitis Functional Index (BASFI), Bath Ankylosing Spondylitis Disease Activity Index (BASDAI), Bath Ankylosing Spondylitis Metrology Index (BASMI) were used. CE training program consisted of 20 exercises: motion and flexibility exercises of the cervical, thoracic, and lumbar spine; stretching of the hamstring muscles, erector spine muscle, and shoulder muscles. IMT training load was based on $50 \%$ of the patient's sustained maximum inspiratory pressure. The patients started by performing ten loaded inspiration with a 60 second rest period between each inspiration for three sets in each session. Four sessions of IMT was applied in a day. The exercises were performed as home program for five days per week, 40 minutes per session. Wilcoxan test was used to compare groups.

Results: After eight weeks follow-up, patients in Group I had a significant increase in PImax $(p=0,000)$, PEmax $(p=0,05)$, and 6MWT $(p=0,041)$ compared with Group II $(p=0,134, p=0,020, p=0,281$, respectively). There were no significant differences of spirometric measurements. Comparison of the groups showed significantly superior results for group I in BASDAI $(p=0,049)$.

Conclusions: Ankylosing spondylitis patients who performed eight weeks of inspiratory muscle training in addition to conventional exercise training, had an increased respiratory muscle strength, a better aerobic capacity, and disease activity than those who performed conventional exercise only. Inspiratory muscle training should be disseminated with these patients due to advantages.

\section{References:}

[1] Dragoi GR, Amaricai E, Dragoi M, Popoviciu H, Avram C. Inspiratory muscle training improves aerobic capacity and pulmonary function in patients with ankylosing spondylitis: A randomized controlled study. 2015 Clinical Rehabilitation, doi: 10.1177/0269215515578292.

Acknowledgements: Inspiratory muscle training should be disseminated with these patients due to advantages.

Disclosure of Interest: None declared

DOI: 10.1136/annrheumdis-2017-eular.6076

\section{SAT0728-HPR A PATIENT SATISFACTION SURVEY: PATIENTS PERCEPTIONS OF BIOLOGIC DOSE TAPERING}

\section{Jones, H. Robinson, D. Rees, V. Jolliffe, J. Trickey, J. Wood, S. Andrews,}

S. Capillas, A. Peall. Rheumatology, Wye Valley NHS Trust, Hereford, UK

Background: Evidence shows that remission can be maintained in selected patients whose biologic medication is dose tapered ${ }^{1}$. In June 2015 we began tapering the doses of selected rheumatoid arthritis, ankylosing spondylitis and psoriatic arthritis patients in disease remission. The process was agreed with the local commissioners and a protocol ${ }^{2}$ was devised to guide safe and evidence based biologic dose tapering. Benefits include reduced medication burden for patients, fewer day-case infusions and financial savings. However, there is a lack of knowledge of patients' perceptions of the process.

Objectives: Our department proposed to investigate patients' perceptions of their experience of dose tapering. We wanted to assess patients' understanding and acceptance of the process and ensure they felt included in decision making. Patient feedback enables reflection and improved practice.

Methods: An anonymous, tick-box, patient satisfaction survey was devised. It requested that patients stated the extent to which they agreed with a statement, also allowing free-text comments. The survey was posted to relevant patients after their identification from our biologics database. A stamped addressed envelope was provided for return of the completed survey.
Results: 56 surveys were posted, $46(82 \%)$ were returned. $31(67 \%)$ of patients were pleased to taper their medication, $35(76 \%)$ felt fully included in decision making and 36 (78\%) agreed that they had the opportunity to ask questions. Of the 13 patients whose disease flared post-taper, $8(62 \%)$ agreed that they were promptly reviewed in clinic, 1 patient $(8 \%)$ disagreed.

Some admitted to anxiety prior to dose tapering, however once established on their new dose, continuing disease remission was reassuring. Patients felt that they could contact the rheumatology helpline if required. Some also expressed that their quality of life had improved due to the decreased medication burden.

$6(37.5 \%)$ of the 16 rituximab patients who replied, disagreed that they were included in the decision to dose taper. Therefore, clinicians must ensure information provided is clear, that a full discussion occurs and the patient has an opportunity to ask questions. On review, it was evident that the wording of the survey may have caused confusion for rituximab patients. The term "dose reduction" may have suggested that the dose of medication in their infusion was reduced. In practice, the patient decreased from two infusions to one.

Conclusions: The majority of patients expressed that they were content with the biologic dose tapering process and felt sufficiently included in decision making. Importantly, a large proportion of patients were of the opinion that they were reviewed quickly when their disease flared.

Practice can be improved from the knowledge that some patients would appreciate more communication regarding the decision to dose taper. Also, when undertaking further patient surveys, questions must be clear and unambiguous.

\section{References:}

[1] JS Smolen, R Landewé, FC Breedveld, et al. EULAR recommendations for the management of rheumatoid arthritis with synthetic and biological diseasemodifying antirheumatic drugs: 2013 update. Ann Rheum Dis 2014;73:492509.

[2] C Holloway. Wye Valley NHS Trust: Biologics in Rheumatology: Dose Tapering Protocol. Sept. 2015.

Disclosure of Interest: None declared

DOI: 10.1136/annrheumdis-2017-eular.1862

\section{SAT0729-HPR PSYCHOLOGICAL PROFILE OF PATIENTS WITH FIBROMYALGIA SYNDROME}

D. Xhaxho $^{1}$, J. Lauri ${ }^{2}$, M.A. Lauri ${ }^{3}$, J. Fleri Soler ${ }^{1}$, A. Borg ${ }^{1}$, C. Mercieca ${ }^{1}$. ${ }^{1}$ Rheumatology, Mater Dei Hospital; ${ }^{2}$ Mathematics; ${ }^{3}$ Psychology, University of Malta, Swatar, Malta

Background: Fibromyalgia (FM) is a chronic non inflammatory condition characterized by pain and fatigue as well as physical and psychological symptoms. Management can be particularly challenging and a combination of pharmacological and non pharmacological treatments are recommended.

Objectives: The aim was to study the level of anxiety and depression in fibromyalgia patients and whether these were associated with symptom severity, functional status, social or demographic factors.

Methods: A cross sectional survey of 155 consecutive patients attending the nurse-led fibromyalgia clinic was carried out. Patients were diagnosed using the 2010 ACR diagnostic criteria. Demographic data, Widespread Pain Index (WPI), Symptom Severity Score (SSS), VAS pain and VAS fatigue were recorded. Patients filled in the self-administered questionnaires including the Revised fibromyalgia impact questionnaire (FIQR), Hospital Anxiety and Depression Scale (HADS). Patients were classified as suffering from high anxiety or depression if HADS was $\geq 11$, moderate if $8-10$ and low $\leq 7$ for each scale respectively.

Cross tabs and chi squared were used to study associations between anxiety and depression and social and demographic factors. Logistic regression analysis was performed to identify whether WPI, SSS, FIQR, VAS pain and VAS fatigue were predictors of severe anxiety and depression.

Results: One hundred fifty five patients ( $92 \%$ female) participated in the survey. The average age was 50.3 years (SD 11.5) and mean duration of symptoms 13.4 years (SD 11.3). The mean HADS-A (anxiety) was 11.8 (SD 4.13), HADS-D (depression) 9.1 (SD 3.8) and FIQR 60.3 (SD 17.6).

High levels of depression were reported by $31.5 \%$, moderate $32.1 \%$ and low $30.3 \%$. High levels of anxiety were reported by $60 \%$, moderate $21.2 \%$ and low $13.3 \%$. Both high levels of anxiety and depression were found in $25.2 \%$ of patients.

SSS was the single best predictor for anxiety $(p=0.001)$ while disease duration $(\mathrm{p}=0.01)$, SSS $(\mathrm{p}=0.02)$ and FIQR $(0.04)$ predicted depression. VAS pain and VAS fatigue were not good predictors of severe anxiety and depression. When patients with high levels of anxiety and depression were compared with those with moderate and lower levels no association was found with age or social factors including occupation, marital status, level of education and family support.

Conclusions: Both severe anxiety and depression were highly prevalent in our FM cohort, particularly anxiety. All FM patients need to be screened for anxiety and depression particularly those with high SSS and FIQR scores and longer disease duration.

A psychiatrist and psychotherapist with a special interest in FM are essential members of the multidisciplinary team to ensure that anxiety and depression are addressed early prior to starting further interventions for FM.

\section{References:}

[1] Jensen KB, Fransson P, Marcus HM, Williams SCR, Choy E, Mainguy Y, Gracely R, Ingvar M, Kosek E (2010) anxiety and depression in fibromyalgia 